\title{
Transport of microRNAs via exosomes
}

Yangxin Li, Zhenya Shen and Xi-Yong Yu

We read with great interest the Review by Boon and Dimmeler (MicroRNAs in myocardial infarction. Nat. Rev. Cardiol. doi:10.1038/nrcardio.2014.207), ${ }^{1}$ who discuss the role of microRNAs (miRNAs) in myocardial infarction and regeneration. Specifically, this Review provides an update on how circulating miRNAs function as communicators between cells using exosomes as shuttle vehicles. An increasing number of studies is enabling the development of novel exosome-based diagnosis and therapies for cardiovascular diseases. ${ }^{2}$ In light of these exciting new developments in exosome research, we wish to highlight some important additional points about the transport of miRNA via exosomes as biomarkers and therapeutic targets.

Circulating miRNAs have been used as biomarkers for many cardiovascular diseases, such as atherosclerosis, heart failure, hypertension, and myocardial infarction. ${ }^{3}$ These miRNAs are packaged either in exosomes or with high-density lipoprotein and are, therefore, quite stable. ${ }^{4}$ Interestingly, although only mature miRNAs have been found in high-density lipoprotein, both pre-miRNAs and mature miRNA have been detected in exosomes. ${ }^{5}$ Exosomes derived from cardiac fibroblasts have been found to contain miRNA passenger strands (known as miRNA star or miRNA $^{*}$ ), which are generally degraded within a cell and induce cardiomyocyte hypertrophy in a paracrine fashion. ${ }^{6}$ The unexpected presence of passenger-strand miRNA in exosomes provides a unique and novel biomarker for cardiovascular disease. Furthermore, different cells might secrete the same miRNA, which makes identifying the source of circulating miRNA difficult. However, exosomes carry markers of the parent cell, which clearly identifies the source of miRNA transferred, thereby enhancing the potential of exosomes as diagnostic biomarkers.?

One of the major challenges of using synthetic miRNA for therapeutic purposes is that exogenously-added miRNA is quickly degraded by the high level of ribonuclease activity in plasma. ${ }^{8}$ Exosomes would, therefore, be ideal candidate carriers, because they are stable and nonimmunogenic., ${ }^{2,9}$ Furthermore, studies have shown that different recipient cells might differ in their capacity to respond to exosomes, with receptor specificity being a crucial factor for internalization of exosomes..$^{10}$ Consequently, the identification of cell-type-specific receptors for targeted delivery of exosomes containing therapeutic miRNA is a necessary step towards successful treatment of specific cardiovascular conditions.

In summary, the transfer of miRNA by exosomes as a method of intercellular communication is a complicated process and many questions remain to be answered. Nevertheless, the Review by Boon and Dimmeler ${ }^{1}$ provides new insights into miRNA-based and exosome-based diagnosis and therapies for cardiovascular diseases.

\section{Institute of Cardiovascular Science} \& Department of Cardiovascular Surgery, First Affiliated Hospital of Soochow University, Suzhou, Jiangsu 215123, People's Republic of China (Y.L., Z.S.). Medical Research Center of Guangdong General Hospital, Guangdong Provincial Cardiovascular Institute, Guangdong Academy of Medical Sciences, Guangzhou, Guangdong 510080, People's Republic of China (X.-Y.Y.). Correspondence to: Y.L. yangxin_li@yahoo.com

\section{Acknowledgements}

The authors were supported by the project for the Priority Academic Program Development of Jiangsu Higher Education Institutions (PAPD) and the National Natural Science Foundation of China (Nos 81120108003 and 81330007).

\section{Competing interests}

The authors declare no competing interests.

1. Boon, R. A. \& Dimmeler, S. MicroRNAs in myocardial infarction. Nat. Rev. Cardiol. http:// dx.doi.org/10.1038/nrcardio.2014.207.

2. Alvarez-Erviti, L. et al. Delivery of siRNA to the mouse brain by systemic injection of targeted exosomes. Nat. Biotechnol. 29, 341-345 (2011).

3. Fichtlscherer, S. et al. Circulating microRNAs in patients with coronary artery disease. Circ. Res. 107, 677-684 (2010).

4. Sahoo, S. \& Losordo, D. W. Exosomes and cardiac repair after myocardial infarction. Circ. Res. 114, 333-344 (2014).

5. Vickers, K. C., Palmisano, B. T., Shoucri, B. M., Shamburek, R. D. \& Remaley, A. T. MicroRNAs are transported in plasma and delivered to recipient cells by high-density lipoproteins. Nat. Cell Biol. 13, 423-433 (2011).

6. Bang, C. et al. Cardiac fibroblast-derived microRNA passenger strand-enriched exosomes mediate cardiomyocyte hypertrophy. J. Clin. Invest. 124, 2136-2146 (2014).

7. Sahoo, S. et al. Exosomes from human CD34+ stem cells mediate their proangiogenic paracrine activity. Circ. Res. 109, 724-728 (2011).

8. Mitchell, P. S. et al. Circulating microRNAs as stable blood-based markers for cancer detection. Proc. Natl Acad. Sci. USA 105, 10513-10518 (2008).

9. Valadi, H. et al. Exosome-mediated transfer of mRNAs and microRNAs is a novel mechanism of genetic exchange between cells. Nat. Cell Biol. 9, 654-659 (2007).

10. Christianson, H. C., Svensson, K. J., van Kuppevelt, T. H., Li, J. P. \& Belting, M. Cancer cell exosomes depend on cell-surface heparan sulfate proteoglycans for their internalization and functional activity. Proc. Natl Acad. Sci. USA 110, 17380-17385 (2013). 